\title{
HLTF suppresses the migration and invasion of colorectal cancer cells via TGF-ß/SMAD signaling in vitro
}

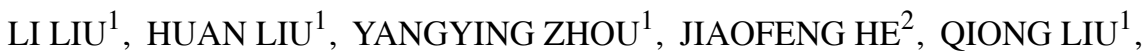 \\ JIAN WANG ${ }^{1}$, MANTING ZENG $^{1}$, DAN YUAN ${ }^{3}$, FENGBO TAN $^{4}$, \\ YUAN ZHOU ${ }^{4}$, HAIPING PEI ${ }^{4}$ and HONG $\mathrm{ZHU}^{1}$ \\ ${ }^{1}$ Department of Oncology, Xiangya Hospital, Central South University; ${ }^{2}$ Department of Oncology, \\ Hunan Academy of Traditional Chinese Medicine Affiliated Hospital, Changsha, Hunan 410008; \\ ${ }^{3}$ Department of Oncology, Zhuzhou No. 2 Hospital, Zhuzhou, Hunan 412005; \\ ${ }^{4}$ Department of Gastrointestinal Surgery, Xiangya Hospital, \\ Central South University, Changsha, Hunan 410008, P.R. China
}

Received May 29, 2018; Accepted September 25, 2019

DOI: 10.3892/ijo.2018.4591

\begin{abstract}
Helicase-like transcription factor (HLTF) has been identified as a tumor suppressor gene. The hypermethylation of HTLF is frequently observed in various types of cancer, including colorectal cancer (CRC). However, the mechanisms through which HLTF suppresses CRC progression remain unclear. Thus, the aim of the present study was to explore the biological function of HLTF in CRC cells and the underlying mechanisms. CRC tissues and cells were used to detect the expression of HLTF. Wound-healing and Transwell assays were performed to assess the motility of CRC cells. The results revealed that HLTF expression was significantly associated with the differentiation status, invasion depth, lymph node metastasis and distant metastasis. A low HLTF expression was significantly associated with a poor survival. Furthermore, HTLF knockdown or ectopic overexpression significantly promoted or suppressed the motility of CRC cells, respectively. With regard to the underlying molecular mechanisms, the protein expression of HTLF was upregulated when the CRC cells were stimulated with transforming growth factor (TGF)- $\beta$, and HLTF upregulation induced an increase in SMAD4 and p-SMAD2/3 expression and a decrease in levels of the TGF- $\beta / \mathrm{SMAD}$ pathway downstream genes, Vimentin and zinc finger e-box binding homeobox 1 (ZEB1). On the whole, the findings of this study suggest that HLTF is negatively associated with the progression of CRC, and its overexpression suppresses
\end{abstract}

Correspondence to: Dr Professor Hong Zhu, Department of Oncology, Xiangya Hospital, Central South University, 87 Xiangya Road, Changsha, Hunan 410008, P.R. China

E-mail: zhuhong0719@126.com

Key words: helicase-like transcription factor, colorectal cancer cells, migration, invasion, transforming growth factor- $\beta / \mathrm{SMAD}$ the migration and invasion of CRC cells by targeting the TGF- $\beta /$ SMAD pathway.

\section{Introduction}

Colorectal cancer (CRC) is one of the most malignant diseases worldwide, with high morbidity and mortality rates. In recent years, early diagnostic techniques and novel therapeutic methods have been used to improve the general outcome of patients with CRC at stages I to IIIB; however, the prognosis remains poor for patients with metastases, with a 5-year survival rate of $<10 \%$ (1). Approximately $50-60 \%$ of patients diagnosed with CRC will develop metastases (2,3). Gaining an understanding of the underlying mechanisms responsible for metastasis and CRC progression is critical in order to improve patient prognosis.

Helicase-like transcription factor (HLTF), which is a member of the SWItch/sucrose non-fermenting (SWI/SNF) family, has been reported to be associated with the prognosis of a number of types of cancer. However, the role of HLTF as a tumor suppressor or promoter remains controversial (4). Previous studies have reported that HLTF functions as a tumor suppressor in DNA repair, genome stability maintenance and gene transcription (4-7). A recent study reported that a high HLTF expression protected cells against apoptosis by impairing the effects of lysosomal autophagy inhibitors (8). The majority of studies on human CRC have focused on the hypermethylation of the HLTF promoter, which is a biomarker of a poor prognosis (9). However, the functional significance and underlying mechanisms of tumor suppression remain unclear.

The transforming growth factor (TGF)- $\beta$ signaling pathway plays a paradoxical role in carcinogens, promoting progression in late CRC $(10,11)$ and inhibiting cell proliferation and apoptosis in early CRC $(12,13)$. Furthermore, it has been reported that TGF- $\beta$ signaling plays a critical role in suppressing tumor metastasis $(14,15)$. Whether TGF- $\beta$ signaling acts as an inhibitor or stimulator depends on the 
function of its core members, including SMAD4. The loss of SMAD4 enhances tumorigenicity and promotes metastasis $(16,17)$.

In this study, to investigate the function of HLTF in CRC cells, we first detected the expression of HLTF in CRC and then investigated the effects of HLTF on the motility of CRC cells. Furthermore, we identified a novel mechanism through which HLTF affects the migration and invasion of CRC cells, by regulating TGF- $\beta /$ SMAD signaling. These findings suggest that HLTF may be a useful prognostic biomarker and a potential target for the diagnosis and treatment of CRC.

\section{Materials and methods}

Clinical specimens and follow-up. A total of 86 para ffin-embedded specimens of primary CRC were obtained by surgical resection at the Department of Pathology, Xiangya Hospital (Changsha, China) between February and December, 2011. The patient clinical data are presented in Table I. Patients were not pre-treated with radiotherapy or chemotherapy prior to surgery. All patients were followed-up until October 30, 2017, and their complete clinical data were collected. Overall survival (OS) is defined as time to death, irrespective of cause, and censored is defined as loss to follow-up. All procedures were performed according to the ethical guidelines of Xiangya Hospital. Informed consent was obtained from all participating patients. For the use of human samples, the protocol for this study was approved by the Xiangya Hospital Ethics Committee (Changsha, China). The 7th edition of the AJCC TNM staging system for CRC was used to classify tumor stage.

Immunohistochemical staining. Sections (5- $\mu \mathrm{m}$-thick) were prepared from the formalin-fixed and paraffin-embedded tissues. Immunohistochemical staining was performed as follows: After dewaxing and rehydrating the tissue slides, antigen retrieval was performed using boiling citrate buffer ( $\mathrm{pH} 6.0$ ) in a pressure cooker for $2.5 \mathrm{~min}$. The slides were subsequently incubated with $3 \% \mathrm{H}_{2} \mathrm{O}_{2}$ for $15 \mathrm{~min}$ at room temperature. Following incubation with rabbit anti-HLTF polyclonal antibody (1:100; ab183042; Abcam, Cambridge, UK) overnight $\left(4^{\circ} \mathrm{C}\right)$, the slides were washed 3 times with PBS to remove excess primary antibodies. The tissues were subsequently incubated with horseradish peroxidase-conjugated anti-mouse/anti-rabbit secondary antibody (cat. no. SPN-9000; undiluted; included in the Biotin-Streptavidin HRP Detection System, Beijing Zhongshan Golden Bridge Biotechnology, Beijing, China) for $30 \mathrm{~min}$ at $37^{\circ} \mathrm{C} \cdot 3,3^{\prime}$-Diaminobenzidine (DAB) was used to visualize positive immune reactions and hematoxylin was used as a counterstain. The primary antibody was omitted for the negative control. Briefly, the sections were scored using a Fourier scale according to the percentage of positive cells and staining intensity: (-) or 0 , tissue specimens without staining $(0-10 \%) ;(+)$ or 1 , tissue specimens with weak staining (10-25\%); (++) or 2, tissue specimens with moderate staining $(25-50 \%)$ and $(+++)$ or 3 , tissue specimens with strong staining $(>50 \%)$. (-) and (+) were defined as low expression, while $(++)$ and $(+++)$ were defined as high expression or overexpression (18). Two board-certified clinical pathologists, who were blinded to the clinical parameters, independently evaluated the staining results. Any disagreement between the two evaluators was resolved by re-evaluation and careful discussion.

Cell culture and treatment. The HCT-8, HCT-116, RKO, SW480 and HT-29 cell lines were obtained from the Cancer Research Institute, Central South University (Changsha, China). LoVo cells were purchased from the Cell Bank of the Chinese Academy of Sciences (Shanghai, China). All cells were cultured in RPMI-1640 medium containing 10\% fetal bovine serum (FBS) and maintained in an incubator at $37^{\circ} \mathrm{C}$ in an atmosphere containing $5 \% \mathrm{CO}_{2}$ (both from Gibco/Thermo Fisher Scientific, Inc., Waltham, MA, USA). The cells were seeded in 6-well plates at a density of $5 \times 10^{4} / \mathrm{cells} / \mathrm{ml}$ and cultured for $24 \mathrm{~h}$ prior to exposure to TGF- $\beta 1$ (cat. no. AF-100-21C-2; Peprotech, Inc., Rocky Hill, NJ, USA) at the concentration of $0,0.5,5 \mathrm{ng} / \mathrm{ml}$. Cells were collected after $48 \mathrm{~h}$ for use in western blot analysis.

RNA extraction and reverse transcription-quantitative $P C R(R T-q P C R)$. TRIzol reagent (Invitrogen/Thermo Fisher Scientific, Inc.) was used to extract total RNA from the cells. A Prime Script RT reagent kit with a gDNA Eraser (Takara Biotechnology Co., Ltd., Dalian, China) was used to perform the reverse transcription. The resulting cDNA was used for qPCR with a CFX96 Real-Time System (Bio-Rad Laboratories, Inc., Hercules, CA, USA) with SYBR Premix Ex Taq II (Takara Biotechnology Co., Ltd.). All processes were performed according to the corresponding manufacturer's instructions. The $2^{-\Delta \Delta C q}$ method (19) was used to calculate the relative abundance of RNA for each gene compared with GAPDH expression. Each reaction was performed in triplicate. The primer sequences used are presented in Table II.

Western blot analysis. The CRC cells were washed and lysed in strong RIPA buffer supplemented with proteinase and phosphatase inhibitors (Thermo Fisher Scientific, Inc.). Total protein was extracted and the concentration was determined by bicinchoninic acid protein assay. Equal amounts of proteins (40 $\mu \mathrm{g}$ per sample) were loaded and separated by $8 \%$ SDS-PAGE. Following electrophoresis, proteins were transferred onto a $0.22 \mu \mathrm{m}$ PVDF membrane (EMD Millipore, Billerica, MA, USA). Following blocking with 5\% non-fat milk, the membranes were incubated with specific primary antibodies at $4{ }^{\circ} \mathrm{C}$ overnight, washed extensively and incubated with secondary antibodies (1:6,000 dilution; cat. no. 7074S; Cell Signaling Technology, Inc., Danvers, MA, USA) for $1 \mathrm{~h}$. The protein bands were visualized and quantified using an ECL Advanced Detection System (EMD Millipore). GAPDH was used as loading control. The primary antibodies used were as follows: HLTF (1:1,000; cat. no. 14286-1-AP; ProteinTech Group, Inc., Chicago, IL, USA), SMAD4 (1:1,000, cat. no. 38454T), SMAD2/3 (1:1,000, cat. no. 8685T), Vimentin (1:1,000, cat. no. 5741T) (all from Cell Signaling Technology, Inc.), p-SMAD2/3 (1:500; cat. no. wl02305), zinc finger e-box binding homeobox 1 (ZEB1; 1:500; cat. no. wl01657; both from Wanleibio, China) and (GAPDH; 1:5,000; cat. no. SAB2701826; Sigma-Aldrich, Merck KGaA, Darmstadt, Germany). 
Table I. Association between HLTF expression and the clinicopathological characteristics of 86 patients with CRC.

\begin{tabular}{lcccc}
\hline & \multicolumn{3}{c}{ HLTF expression } & \\
\cline { 2 - 3 } $\begin{array}{l}\text { Clinicopathological } \\
\text { characteristics }\end{array}$ & $\mathrm{n}$ & Low & High & P-value \\
\hline $\begin{array}{l}\text { Age (years) } \\
<65\end{array}$ & & & & \\
$\geq 65$ & 58 & 36 & 22 & \\
Sex & & & & \\
Male & 45 & 22 & 23 & $0.1755^{\mathrm{a}}$ \\
Female & 41 & 26 & 15 & \\
Tumor differentiation & & & & \\
Well-moderate & 47 & 16 & 31 & $<0.0001^{\mathrm{a}}$ \\
Poor & 39 & 32 & 7 & \\
TNM stage & & & & \\
I-II & 38 & 11 & 27 & $<0.0001^{\mathrm{a}}$ \\
III-IV & 48 & 37 & 11 & \\
Depth of invasion & & & & \\
T1-T2 & 18 & 1 & 17 & $<0.0001^{\mathrm{b}}$ \\
T3-T4 & 68 & 47 & 21 & \\
Lymph node metastasis & & & & \\
N0 & 41 & 13 & 28 & $<0.0001^{\mathrm{a}}$ \\
N1-N2 & 45 & 35 & 10 & \\
Distant metastasis & & & & \\
M0 & 65 & 31 & 34 & $0.0076^{\mathrm{a}}$ \\
M1 & 21 & 17 & 4 & \\
\hline
\end{tabular}

HLTF, helicase-like transcription factor; CRC, colorectal cancer;

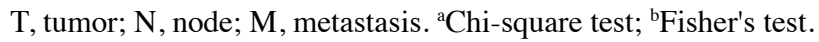

shRNAs, siRNA and vector transfection. shRNAs targeting HLTF and a control shRNA were purchased from GeneChem (Shanghai, China). The shRNA vector (GV493), also from GeneChem, was hU6-MCS-CBh-gcGFP-IRES-puromycin. Moreover, the shRNAs obtained targeted the following sequences of HTLF (NM_139048): shRNA 1\# targets AGGT GGAGTTGGTTTGAAT and shRNA 2\# targets TATTAGAG AACCGGCCTTA. The HCT-8 and HCT-116 cells were infected with the purified lentiviruses containing the shRNA sequence targeting HLTF or the control sequence lentivirus for $24 \mathrm{~h}$. Stably transfected cells were isolated using $2 \mu \mathrm{g} / \mathrm{ml}$ puromycin (Sigma; Merck KGaA) for 7 days. In addition, human SMAD4 siRNA was purchased from GenePharma (Shanghai, China). The SMAD4-siRNA sequences were as follows: sense, 5'-GAGAAGTTCTCAAAGTTAA-3' and antisense, 5'-TTAACTTTGAGAACTTCTC-3'. The pGV141-HLTF plasmid (purchased from GeneChem), is an expression vector, containing HLTF cDNA and the structure of the vector is CMV-MCS-3FLAG-SV40-Neomycin. When the cells are transfected with pGV141-HLTF, they then overexpress HLTF. ViaFect ${ }^{\mathrm{TM}}$ Transfection reagent (cat. no. E4981; Promega Corp., Madison, WI, USA) was used to transfect the plasmid into RKO cells according to the manufacturer's instructions.
Table II. Sequences of the primers for reverse transcription-quantitative polymerase chain reaction.

\begin{tabular}{ll}
\hline Gene & \multicolumn{1}{c}{ Sequence } \\
\hline HLTF & F: 5'-GCTTAGACGGTTCCATGGCTCAAA-3' \\
& R:5'-TCCAGGCTGGGTCCATTAAGAACA-3' \\
SMAD4 & F: 5'-CTCATGTGATCTATGCCCGTC-3' \\
& R: 5'-AGGTGATACAACTCGTTCGTAGT-3' \\
GAPDH & F: 5'-CCCTCAAGATTGTCAGCAATG-3' \\
& R: 5'-GTCCTCAGTGTAGCCCAGGAT-3' \\
\hline
\end{tabular}

HLTF, helicase-like transcription factor; SMAD4, Caenorhabditis elegans SMA and Drosophila MAD 4; GAPDH, glyceraldehyde-3-phosphate dehydrogenase; F, forward; R, reverse.

The siRNA and plasmid were co-transfected into the cells using Lipofectamine ${ }^{\circledR} 2000$ reagent (cat. no. 11668027; Invitrogen; Thermo Fisher Scientific, Inc.).

Wound healing assay. The cells were cultured on 6-well plates with RPMI-1640 containing 10\% FBS. When the cell density reached $70-80 \%$, the bottom of the plate was scratched with a $100 \mu \mathrm{l}$ pipette tip to create a cell-free gap, following which the cells were incubated for 48 (HCT-116 and RKO) or $72 \mathrm{~h}$ (HCT-8) with RPMI-1640 medium containing 1\% FBS. An inverted Olympus IX50 microscope (Olympus Corp., Tokyo, Japan) was used to obtain phase-contrast images of the wound healing process at different time-points after scratching. The size of the healed wound was then compared with the size of the initial wound.

Transwell assay. A total of $5 \times 10^{4}$ cells suspended in $100 \mu 1$ serum-free RPMI-1640 medium were seeded into the upper chamber of a Transwell apparatus (Corning Inc., Corning, NY, USA; $8 \mu \mathrm{m}$ pore) with $50 \mu \mathrm{l}$ Matrigel (BD Biosciences, Franklin Lakes, NJ, USA). A total of $600 \mu 1$ RMPI-1640 medium containing $10 \%$ FBS was added to the lower chamber. Following incubation at $37^{\circ} \mathrm{C}$ for $48 \mathrm{~h}$ (HCT-116 and RKO) or $60 \mathrm{~h}$ (HCT-8), cells in the upper chamber were removed with a cotton swab and cells on the lower surface were fixed in $1 \%$ paraformaldehyde followed by staining with $0.1 \%$ crystal violet solution (Beyotime Institute of Biotechnology, Haimen, China) at room temperature. The number of invading cells was determined for 5 randomly selected fields (x200 magnificatoin) under a microscope (Leica inverted microscope DMi1; Leica, Wetzlar, Germany). Three independent experiments were performed and the mean was calculated.

Prediction of transcription factor binding sites. GCBI online software (https://www.gcbi.com.cn/gclib/html/index) was used to predict the putative binding sites for HLTF on SMAD4 promoter region.

Statistical analysis. Data were analyzed using GraphPad Prism 5.0 software (GraphPad Software, Inc., La Jolla, CA, USA). Differences between 2 groups were assessed using 
Stage I
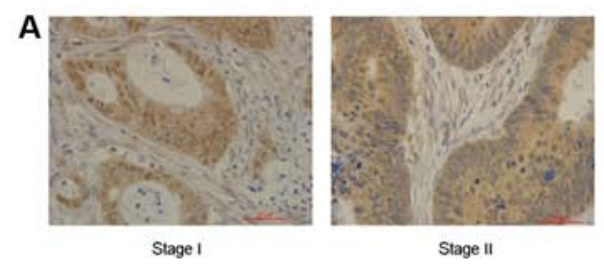

C

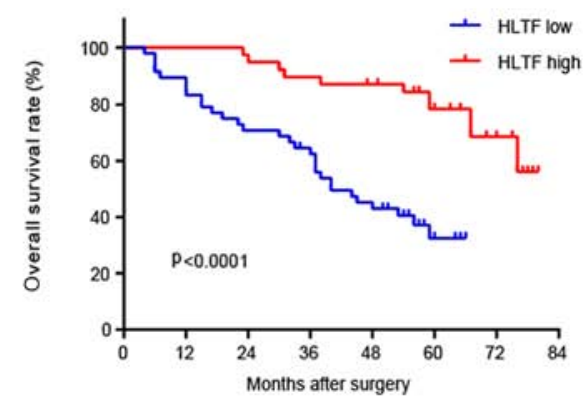

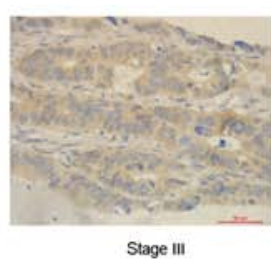

D

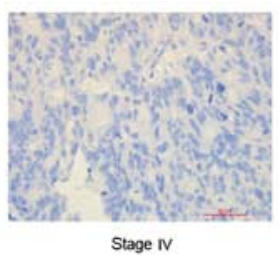

Stage IV
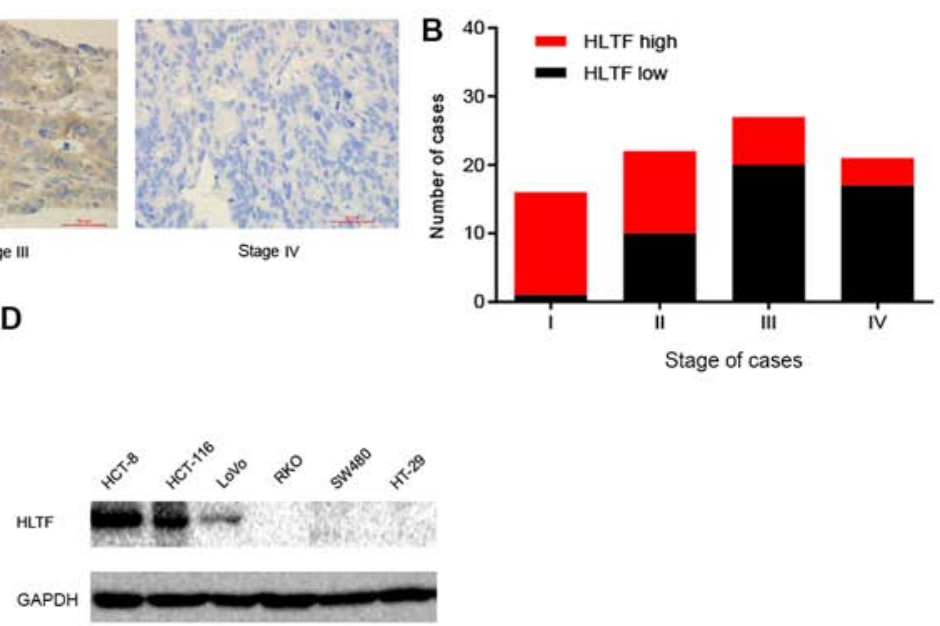

Figure 1. HLTF expression is negatively associated with the progression of CRC. (A and B) Immunohistochemical HLTF staining of CRC tissues scored stage I-IV obtained from patients shown in Table I. Magnification, $\mathrm{x} 400$. Scale bar, $50 \mu \mathrm{m}$. HLTF was expressed at higher levels in patients with early-stage CRC compared to patients with late-stage CRC. (C) Survival plot of 86 patients with CRC. HLTF expression was negatively associated with the overall survival time. (D) HLTF protein immunodetection by western blot analysis prepared with whole cell extracts (see Materials and methods). HLTF, helicase-like transcription factor; CRC, colorectal cancer.

a Student's t-test. For 3 or more groups, one-way ANOVA test was used followed by Tukey's post hoc test for multiple comparisons. The associations between HLTF expression and the patient clinicopathological characteristics were determined using the Chi-square and Fisher's exact tests. Overall survival (OS) curves were plotted according to the Kaplan-Meier method, and differences in survival were examined using the log-rank test. $\mathrm{P}<0.05$ was considered to indicate a statistically significant difference.

\section{Results}

HLTF expression is negatively associated with the progression of CRC. To verify the expression of HLTF in CRC, immunochemistry was performed on CRC tissues at various stages. The results revealed that HLTF expression decreased with the increasing TNM stage (Fig. 1A and B). This suggests that HLTF expression is negatively correlated with CRC progression. To explore the association between HLTF and CRC, we analyzed the association between HLTF expression and the patient clinicopathological characteristics. As shown in Table I, the expression of HLTF was significantly associated with the differentiation status $(\mathrm{P}<0.0001)$, TNM stage $(\mathrm{P}<0.0001)$, depth of invasion $(\mathrm{P}<0.0001)$, lymph node metastasis $(\mathrm{P}<0.0001)$ and distant metastasis $(\mathrm{P}<0.01)$. However, no significant association was observed between HLTF expression and age $(\mathrm{P}=0.0927)$ or sex $(\mathrm{P}=0.1755)$. To further assess the prognostic value of HLTF in CRC, we performed a survival analysis. Kaplan-Meier analysis revealed that patients with CRC with a high HLTF expression had a significantly longer OS compared with patients with a low HLTF expression (Fig. 1C, log-rank test, $\mathrm{P}<0.0001$ ), suggesting that a low HLTF protein expression contributes to CRC progression and is associated with a poor prognosis. Based on these results, we thus concluded that the expression of HLTF is negatively associated with the progression of CRC.
HLTF knockdown in CRC cells promotes cell migration and invasion. The HCT- 8 and HCT-116 cell lines were used to assess the effects of HLTF on cell migration and invasion due to their high expression of HLTF (Fig. 1D). We manipulated the cells by knocking down HLTF (Fig. 2A). The results of wound healing $(\mathrm{P}<0.01$; Fig. 2C) and Transwell assays $(\mathrm{P}<0.0001$; Fig. 2B) demonstrated that HLTF knockdown significantly enhanced the migration and invasion of CRC cells in vitro.

HLTF overexpression in RKO cells suppresses cell migration and invasion. HLTF was overexpressed in RKO cells to assess whether the migration and invasion abilities would decrease. Evidence of HLTF overexpression in RKO cells is shown in Fig. 3A. HLTF upregulation significantly suppressed the migration $(\mathrm{P}<0.0001$; Fig. 3B) and invasion $(\mathrm{P}<0.0001$; Fig. 3C) of the CRC cells. These results confirmed that HLTF overexpression suppressed the migration and invasion of CRC cells in vitro.

HLTF affects the TGF- $\beta / S M A D$ pathway in CRC cells. In order to elucidate the mechanisms through which HLTF suppresses the migration and invasion of CRC cells, we investigated metastasis-related pathways. The results of western blot analysis revealed that the expression of HLTF, SMAD4 and p-SMAD2/3, but not that of total SMAD2/3 were increased when the HCT-8, HCT-116 and RKO cells were stimulated with TGF- $\beta 1$ (Fig. 4A), suggesting that HLTF is regulated by the TGF- $\beta$ signaling pathway. We also investigated the mechanism through which HLTF affects the TGF- $\beta$ signaling pathway. As shown in Fig. 4B, we observed that HLTF knockdown decreased the expression of SMAD4 and p-SMAD2/3, but not that of total SMAD2/3 in both the HCT-8 and HCT-116 cells. The results of RT-qPCR confirmed that the mRNA expression of SMAD4 was also decreased (Fig. 4D). In addition, when using GCBI online software, we predicted that the SMAD4 gene promoter presented putative binding 
A

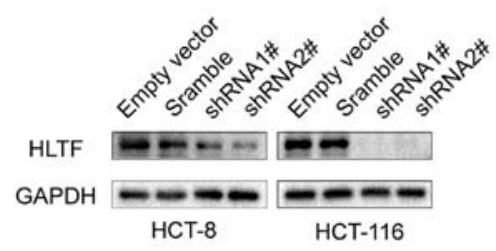

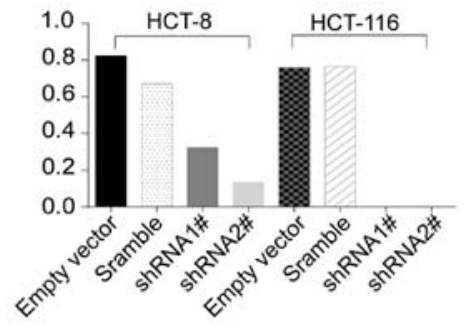

B

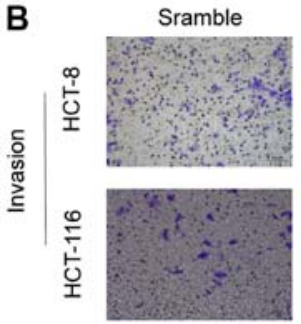
shRNA1\#

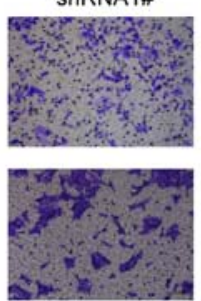
ShRNA2\#
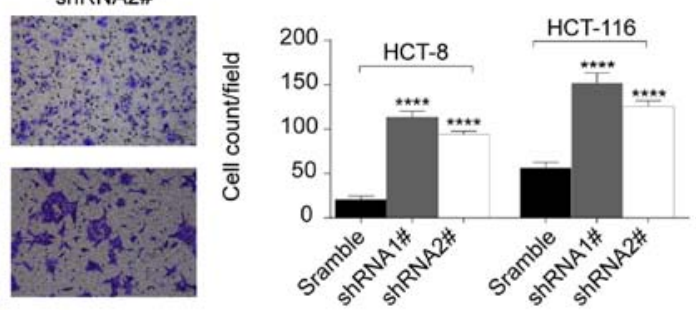

C
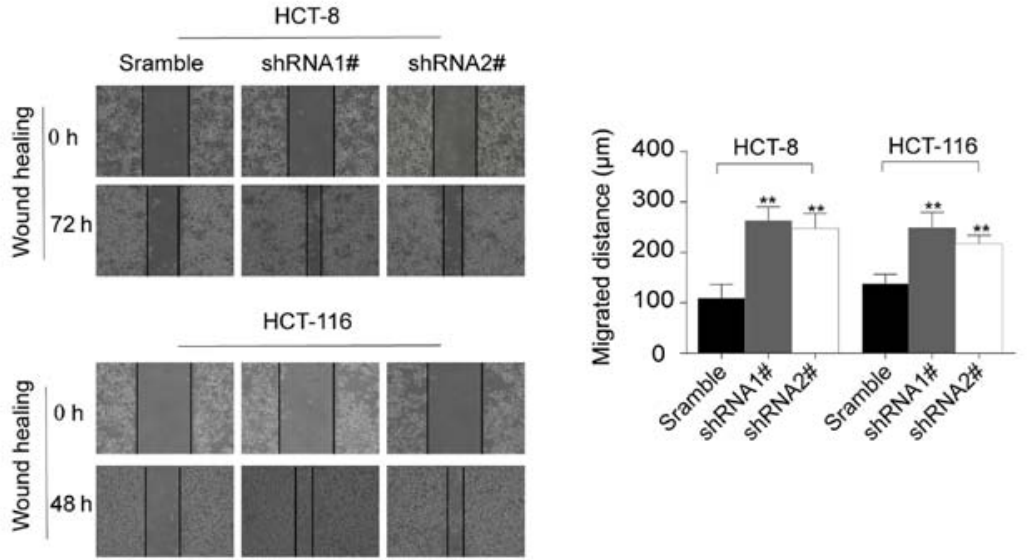

Figure 2. A low HLTF expression promotes the migration and invasion of CRC cells. (A) HLTF was knockdown in both HCT-8 and HCT-116 cells. HLTF protein level in CRC cells when cells were transfected with empty vector, scramble control vector, shRNA1\# and shRNA2\#. (B) A Matrigel assay revealed that HLTF knockdown enhanced the invasion of HCT- 8 and HCT-116 cells $\left({ }^{* * * *} \mathrm{P}<0.0001\right.$ vs. scramble). (C) A wound healing assay revealed that HLTF knockdown enhanced the migration of the HCT-8 and HCT-116 cells ( ${ }^{* *} \mathrm{P}<0.01$ vs. scramble). HLTF, helicase-like transcription factor; CRC, colorectal cancer.

A

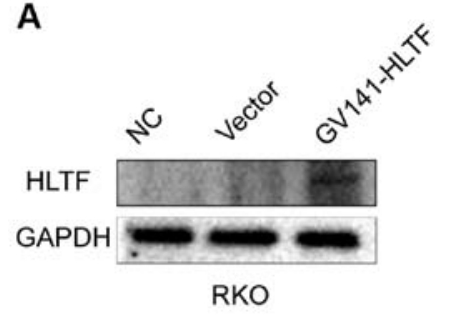

C

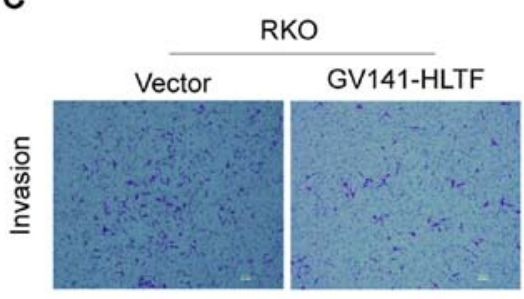

B
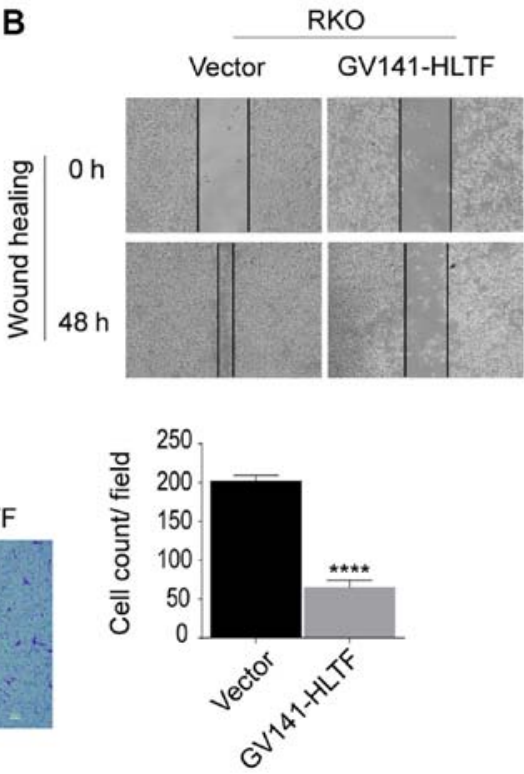

RKO

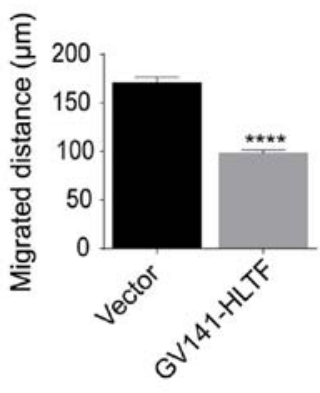

RKO

Figure 3. HLTF upregulation in RKO cells suppresses cell migration and invasion. (A) HLTF protein level in RKO cells when cells were transfected with no vector (NC), control vector and GV141-HLTF. Wound healing assay and Transwell assay revealed that HLTF upregulation suppresses the (B) migration and (C) invasion of the RKO cells $\left({ }^{* * * *} \mathrm{P}<0.0001\right.$ vs. vector). HLTF, helicase-like transcription factor. 
A

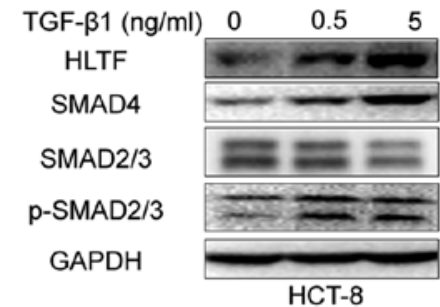

B

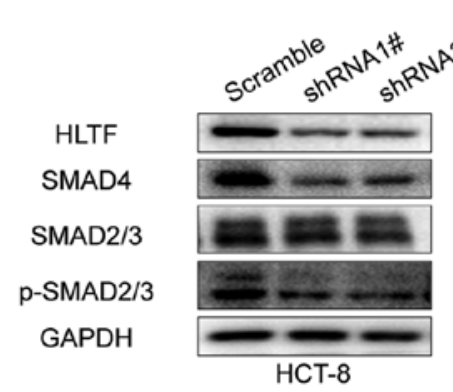

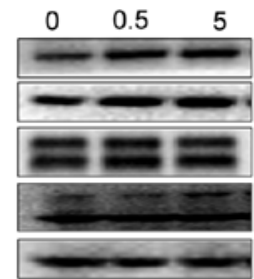

HCT-116

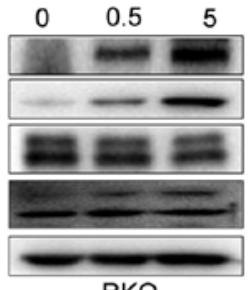

RKO

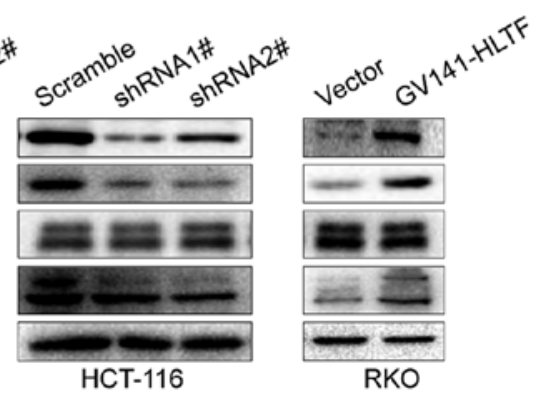

C
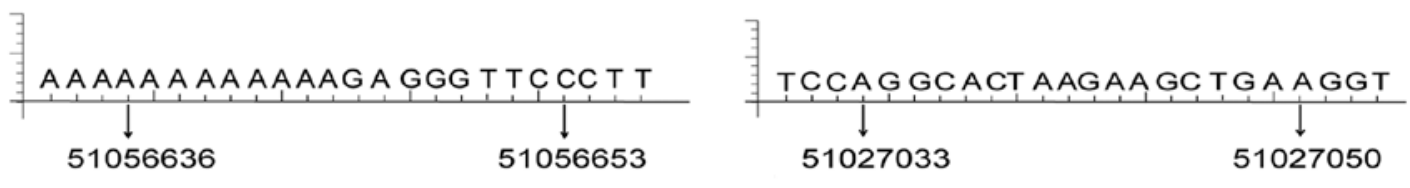

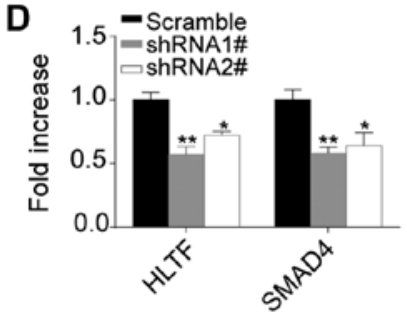

HCT-8

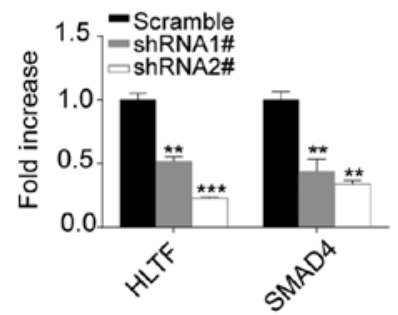

HCT-116

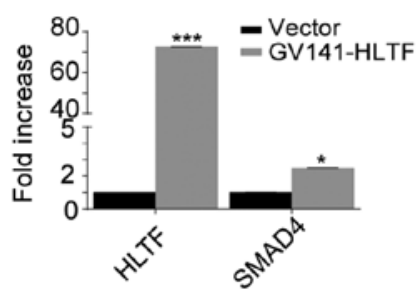

RKO

E

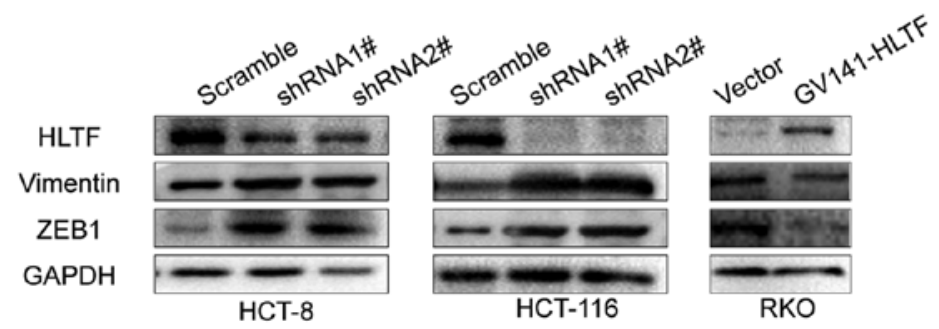

Figure 4. HLTF expression is associated with TGF- $\beta /$ SMAD signaling. (A) HLTF protein level increased in CRC cells following stimulation with $0,0.5$ or $5 \mathrm{ng} / \mathrm{ml}$ TGF- $\beta 1$. (B) HLTF knockdown in HCT-8 and HCT-116 cells reduced SMAD4 and p-SMAD2/3 expression, whereas HLTF upregulation in RKO cells increased SMAD4 and p-SMAD2/3 expression. The total SMAD2/3 level was not significantly affected in the HCT-8, HCT-116 and RKO cells. (C) Predicted sequences of putative binding sites for HLTF on SMAD4 promoter region by using GCBI online software. Left panel: aaaaAAAAAgagggttc, the region band from beginning to end is 51056636 - 51056653; right panel: aggcACTAAgaagctga, the region band from beginning to end is 51027033 - 51027050 . (D) Reverse transcription-quantitative polymerase chain reaction conformed that the SMAD4 mRNA levels were reduced in the HCT-8 and HCT-116 cells following HLTF knockdown ( $\mathrm{P}<0.05$ and ${ }^{* *} \mathrm{P}<0.01$ vs. scramble), while they were increased in the HLTF-RKO cells ( $\mathrm{P}<0.05$ vs. vector). (E) HLTF knockdown promoted the expression of Vimentin and ZEB1 protein, whereas HLTF overexpression had the opposite effect. HLTF, helicase-like transcription factor; CRC, colorectal cancer, TGF- $\beta$, transforming growth factor- $\beta$.

sites for HLTF (51056636 - 51056653, aaaAAAAAgagggttc; 51027033 - 51027050, aggcACTAAgaagctga) (Fig. 4C). Furthermore, in the HCT- 8 and HCT-116 cells, HLTF knockdown upregulated the expression of Vimentin and ZEB1, which are downstream targets of TGF- $\beta$ signaling (Fig. 4E). The opposite result was observed in the RKO cells overexpressing HLTF (Fig. 4B, D and E). These results suggest that HLTF is associated with the TGF- $\beta$ /SMAD pathway, and that a high HLTF expression suppresses the migration and invasion of CRC cells by targeting TGF- $\beta /$ SMAD signaling.
HLTF suppresses tumor aggressiveness through TGF- $\beta / S M A D$ signaling. To confirm whether HLTF affects tumor aggressiveness via the TGF- $\beta /$ SMAD pathway, SMAD4 was knocked down using siRNA in HLTF-transduced RKO (HLTF-siSMAD4-RKO) cells. With 3 siRNAs targeting SMAD4, we finally selected the most effective siRNA (siRNA1\#) for the following experiments (data not shown). As shown in Fig. 5A, the SMAD4 mRNA level was significantly decreased in the HLTF-siSMAD4-RKO cells compared with the HLTF-RKO cells $(\mathrm{P}<0.01)$. Furthermore, the 


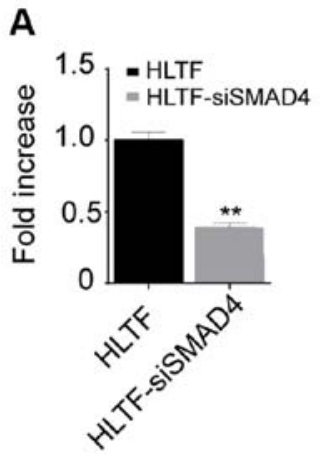

RKO
B

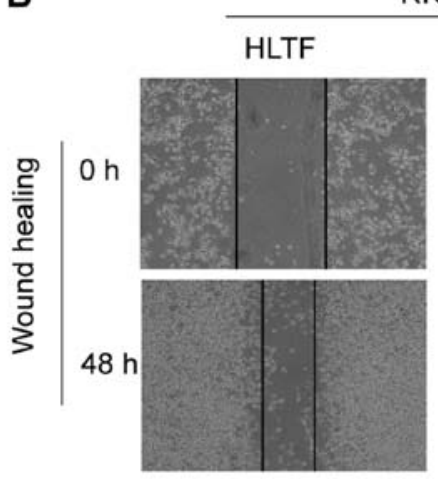

RKO
RKO
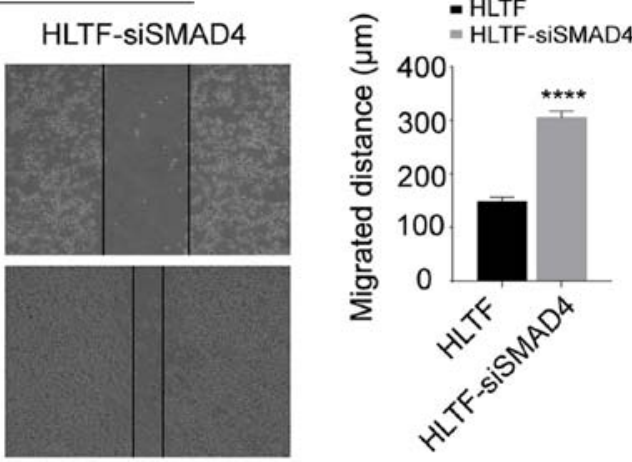

RKO

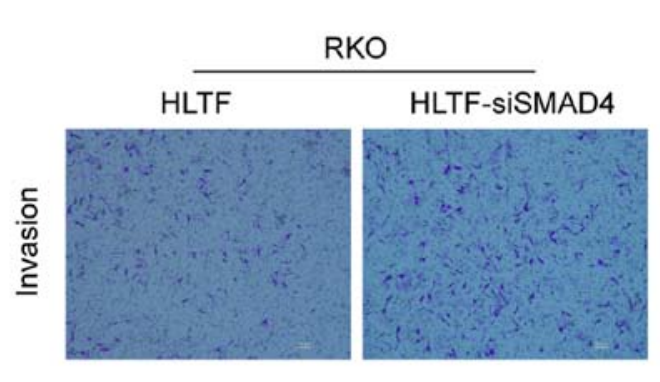

\section{C}

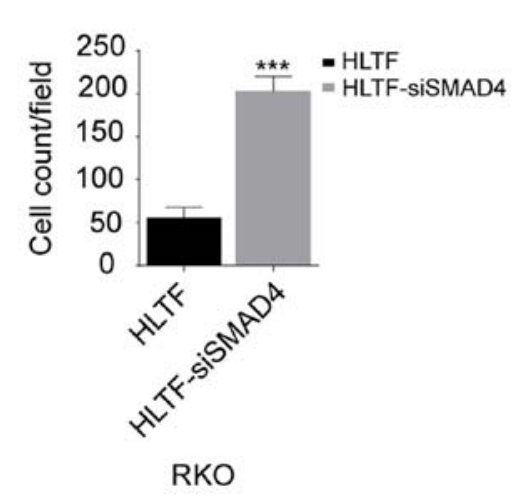

Figure 5. HLTF suppresses CRC cell migration and invasion via TGF- $\beta$ /SMAD signaling. (A) Reverse transcription-quantitative polymerase chain reaction confirmed that the SMAD4 mRNA levels were reduced in RKO cells following co-transfection with HLTF plasmid and siSMAD4 (** $<0.01$ vs. HLTF), (B and C) The wound healing and Matrigel assays revealed that the migratory and invasive ability was higher in the HLTF-siSMAD4-RKO cells compared with the HLTF-RKO cells $\left({ }^{* * *} \mathrm{P}<0.001\right.$ and ${ }^{* * * *} \mathrm{P}<0.0001$ vs. HLTF).

results of wound healing $(\mathrm{P}<0.0001$; Fig. 5B) and Transwell assays $(\mathrm{P}<0.001$; Fig. $5 \mathrm{C})$ revealed that the motility of the HLTF-siSMAD4-RKO cells was increased compared with that of the HLTF-RKO cells. These results further support the conclusion that HLTF affects CRC cells via the TGF- $\beta$ /SMAD signaling pathway.

\section{Discussion}

The role of HLTF in cancer progression is unclear; previous studies have stated that it functions as an oncogene or tumor suppressor. In the kidneys, hypopharynx and cervical cancer, HLTF serves as an oncogene (20-23), while in the thyroid and digestive carcinoma, HLTF functions as a tumor suppressor $(24,25)$. Furthermore, the functional loss of HLTF promotes intestinal carcinogenesis (26). In CRC, promoter hypermethylation is frequently observed (27-29). It is significantly associated with tumor stage, metastatic disease $(30,31)$ and is regarded as a biomarker of poor prognosis $(32,33)$ in CRC. However, the potential mechanisms through which HLTF functions in CRC have not yet been reported. This study revealed an association between HLTF expression and metastasis in CRC. Mechanistic studies have revealed that HLTF suppresses the motility of CRC cells via the TGF- $\beta$ signaling pathway.

In 2016, Cheng et al demonstrated that HLTF expression was reduced in adult acute myeloid leukemia patients with more aggressive disease phenotypes (34). In this study, the results of immunohistochemistry revealed that HLTF expression was negatively associated with the differentiation status, tumor invasive depth, lymph metastasis and distant metastasis in CRC. A poor differentiation status, lymph node and distant metastasis have been reported to be negative prognostic factors in CRC $(35,36)$. These results suggest that CRC tissues with a low HLTF expression are more aggressive and are associated with severe cancer progression. Survival analysis revealed that a low HLTF expression was associated with a poor prognosis. Metastasis is characterized by a series of pathological events, including deep invasion into the intestinal wall and the lymphovascular or circulatory systems (37). We then examined the motility of CRC cells when HLTF was knocked down or overexpressed. The results indicated that silencing HLTF markedly enhanced the migration and invasion of HCT-8 and HCT-116 cells, while HLFT overexpression suppressed the the migratory and invasive ability of the RKO cells in vitro. From these results, it can be concluded that HLTF is a biomarker of CRC aggressiveness.

There is evidence to indicate that HLTF functions as a tumor suppressor mainly through two mechanisms: Promoter hypermethylation or alternative mRNA splicing (4). The results of the present study provide novel insight into the suppressive role of HLTF in CRC. Firstly, a previous study employing a MetaCoret ${ }^{\mathrm{TM}}$ enrichment pathway analysis suggested that HLTF is closely asscociated with cell adhesion and TGF- $\beta$ signaling (38). Our study showed that TGF- $\beta$ stimulation induced HLTF overexpression in CRC cells. In addition, HLTF 
knockdown in HCT-8 and HCT-116 cells resulted in a decrease in SMAD4 and p-SMAD2/3 expression and a reduction in the expression of their downstream targets, Vimentin and ZEB1. The opposite was observed in HLTF-RKO cells.

SMAD4, which is a CRC suppressor, is an important factor in TGF- $\beta$ signaling. SMAD4 mutation or loss can strongly affect TGF- $\beta /$ SMAD signaling and promote CRC cell metastasis (17,39-42). There is evidence to indicate that the loss of SMAD4 promotes CRC cell metastasis $(43,44)$. Furthermore, the expression of SMAD4 is positively associated with survival in colon cancer (45). In addition, we predicted that the SMAD4 gene promoter presented putative binding sites for HLTF using GCBI online software. The results of this study indicated that HLTF depletion reduced the expression of SMAD4 and p-SMAD2/3 in HCT-8 and HCT-116 cells, while their expression was increased in the HLTF-RKO cells. Furthermore, transfection with siRNA targeting SMAD4 promoted cell motility. We therefore concluded that HLTF inhibits the migration and invasion of CRC cells via the TGF- $\beta$-HLTF-SMAD signaling pathway, and we hypothesize that HLTF may be a direct transcriptional activator of the SMAD4 gene. However, further investigation is required to elucidate the detailed mechanisms.

Evidence indicate that both HLTF and TGF- $\beta$ signaling play a paradoxical role in cancer progression $(4,11)$. Whether TGF- $\beta$-HLTF-SMAD signaling plays a similar role in CRC is unclear. Further experiments in vivo and the analysis of clinical samples are required for a more in depth investigation.

In conclusion, the results of the present study suggest that HLTF functions as a tumor suppressor gene in CRC. HLTF downregulation promotes the migration and invasion of CRC cells in vitro. Furthermore, HLTF expression is negatively associated with OS in patients with CRC. HLTF may suppress the migration and invasion of CRC cells by targeting TGF- $\beta$ /SMAD signaling. Taken together, these results suggest that HLTF may be a potential prognostic biomarker for CRC.

\section{Acknowledgements}

Not applicable.

\section{Funding}

This study was supported by the Natural Science Foundation of China (nos. 81372428, and 81702956).

\section{Availability of data and materials}

All data generated or analyzed during this study are included in this published article or are available from the corresponding author on reasonable request.

\section{Authors' contributions}

HZ and FT conceived and designed the study. LL, HL, YaZ, $\mathrm{JH}$ and QL performed the experiments. LL drafted the manuscript. MZ and JW revised the manuscript. DY collected the clinical data. MZ, JW, YuZ and HPP participated in data analysis and interpretation of the results. All authors have read and approved the final manuscript.

\section{Ethics approval and consent to participate}

Informed consent was obtained from all participating patients. For the use of human samples, the protocol for this study was approved by the Xiangya Hospital Ethics Committee (Changsha, China).

\section{Patient consent for publication}

Not applicable.

\section{Competing interests}

The authors declare that they have no competing interests.

\section{References}

1. Siegel RL, Miller KD and Jemal A: Cancer statistics, 2016. CA Cancer J Clin 66: 7-30, 2016.

2. Van Cutsem E, Nordlinger B, Adam R, Köhne CH, Pozzo C, Poston G, Ychou M and Rougier P; European Colorectal Metastases Treatment Group: Towards a pan-European consensus on the treatment of patients with colorectal liver metastases. Eur J Cancer 42: 2212-2221, 2006.

3. Lee WS, Yun SH, Chun HK, Lee WY, Yun HR, Kim J, Kim K and Shim YM: Pulmonary resection for metastases from colorectal cancer: Prognostic factors and survival. Int J Colorectal Dis 22: 699-704, 2007.

4. Dhont L, Mascaux C and Belayew A: The helicase-like transcription factor (HLTF) in cancer: Loss of function or oncomorphic conversion of a tumor suppressor? Cell Mol Life Sci 73: 129-147, 2016.

5. Poole LA and Cortez D: Functions of SMARCAL1, ZRANB3, and HLTF in maintaining genome stability. Crit Rev Biochem Mol Biol 52: 696-714, 2017.

6. Kile AC, Chavez DA, Bacal J, Eldirany S, Korzhnev DM, Bezsonova I, Eichman BF and Cimprich KA: HLTF's ancient HIRAN domain binds 3' DNA ends to drive replication fork reversal. Mol Cell 58: 1090-1100, 2015.

7. Taglialatela A, Alvarez S, Leuzzi G, Sannino V, Ranjha L, Huang JW, Madubata C, Anand R, Levy B, Rabadan R, et al: Restoration of replication fork stability in BRCA1- and BRCA2-deficient cells by inactivation of SNF2-family fork remodelers. Mol Cell 68: 414-430.e8, 2017.

8. Piao S, Ojha R, Rebecca VW, Samanta A, Ma XH, Mcafee Q, Nicastri MC, Buckley M, Brown E, Winkler JD, et al: ALDH1A1 and HLTF modulate the activity of lysosomal autophagy inhibitors in cancer cells. Autophagy 13: 2056-2071, 2017.

9. Debauve G, Capouillez A, Belayew A and Saussez S: The helicase-like transcription factor and its implication in cancer progression. Cell Mol Life Sci 65: 591-604, 2008.

10. Tauriello DVF and Batlle E: Targeting the microenvironment in advanced colorectal cancer. Trends Cancer 2: 495-504, 2016.

11. Drabsch Y and ten Dijke P: TGF- $\beta$ signalling and its role in cancer progression and metastasis. Cancer Metastasis Rev 31: 553-568, 2012.

12. Massagué J: TGF $\beta$ signalling in context. Nat Rev Mol Cell Biol 13: 616-630, 2012.

13. Wakefield LM and Hill CS: Beyond TGF $\beta$ : Roles of other TGF $\beta$ superfamily members in cancer. Nat Rev Cancer 13: 328-341, 2013.

14. Yang L, Liu Z, Tan J, Dong $H$ and Zhang X: Multispectral imaging reveals hyper active TGF- $\beta$ signaling in colorectal cancer. Cancer Biol Ther 19: 105-112, 2018.

15. Fritzmann J, Morkel M, Besser D, Budczies J, Kosel F, Brembeck FH, Stein U, Fichtner I, Schlag PM and Birchmeier W; A colorectal cancer expression profile that includes transforming growth factor beta inhibitor BAMBI predicts metastatic potential. - PubMed - NCBI. Gastroenterology 137: 165-175, 2009.

16. Papageorgis P, Cheng K, Ozturk S, Gong Y, Lambert AW, Abdolmaleky HM, Zhou JR and Thiagalingam S: Smad4 inactivation promotes malignancy and drug resistance of colon cancer. Cancer Res 71: 998-1008, 2011. 
17. Zhang B, Halder SK, Kashikar ND, Cho YJ, Datta A, Gorden DL and Datta PK: Antimetastatic role of Smad4 signaling in colorectal cancer. Gastroenterology 138: 969-80.e1, 3, 2010.

18. Rizzardi AE, Johnson AT, Vogel RI, Pambuccian SE, Henriksen J, Skubitz AP, Metzger GJ and Schmechel SC: Quantitative comparison of immunohistochemical staining measured by digital image analysis versus pathologist visual scoring. Diagn Pathol 7: 42, 2012

19. Livak KJ and Schmittgen TD: Analysis of relative gene expression data using real-time quantitative PCR and the 2(-Delta Delta C(T)) Method. Methods 25: 402-408, 2001.

20. Debauve G,Nonclercq D, RibaucourF, Wiedig M, Gerbaux C,LeoO, Laurent G, Journé F, Belayew A and Toubeau G: Early expression of the Helicase-Like Transcription Factor (HLTF/SMARCA3) in an experimental model of estrogen-induced renal carcinogenesis. Mol Cancer 5: 23, 2006

21. Capouillez A, Decaestecker C, Filleul O, Chevalier D, Coppée F, Leroy X, Belayew A and Saussez S: Helicase-like transcription factor exhibits increased expression and altered intracellular distribution during tumor progression in hypopharyngeal and laryngeal squamous cell carcinomas. Virchows Arch 453: 491-499, 2008.

22. Capouillez A, Debauve G, Decaestecker C, Filleul O, Chevalier D, Mortuaire G, Coppée F, Leroy X, Belayew A and Saussez S: The helicase-like transcription factor is a strong predictor of recurrence in hypopharyngeal but not in laryngeal squamous cell carcinomas. Histopathology 55: 77-90, 2009.

23. Ye C, Sun NX, Ma Y, Zhao Q, Zhang Q, Xu C, Wang SB, Sun SH, Wang F and Li W: MicroRNA-145 contributes to enhancing radiosensitivity of cervical cancer cells. FEBS Lett 589: 702-709, 2015.

24. Arcolia V, Paci P, Dhont L, Chantrain G, Sirtaine N, Decaestecker C, Remmelink M, Belayew A and Saussez S: Helicase-like transcription factor: A new marker of well-differentiated thyroid cancers. BMC Cancer 14: 492, 2014.

25. Kim JJ, Chung SW, Kim JH, Kim JW, Oh JS, Kim S, Song SY, Park J and Kim DH: Promoter methylation of helicase-like transcription factor is associated with the early stages of gastric cancer with family history. Ann Oncol 17: 657-662, 2006.

26. Sandhu S, Wu X, Nabi Z, Rastegar M, Kung S, Mai S and Ding H: Loss of HLTF function promotes intestinal carcinogenesis. Mol Cancer 11: 18, 2012.

27. Hibi K, Nakayama H, Kanyama Y, Kodera Y, Ito K, Akiyama S and Nakao A: Methylation pattern of HLTF gene in digestive tract cancers. Int J Cancer 104: 433-436, 2003.

28. Borinstein SC, Conerly M, Dzieciatkowski S, Biswas S, Washington MK, Trobridge P, Henikoff S and Grady WM: Aberrant DNA methylation occurs in colon neoplasms arising in the azoxymethane colon cancer model. Mol Carcinog 49: 94-103, 2010.

29. Moinova HR, Chen WD, Shen L, Smiraglia D, Olechnowicz J, Ravi L, Kasturi L, Myeroff L, Plass C, Parsons R, et al: HLTF gene silencing in human colon cancer. Proc Natl Acad Sci USA 99: 4562-4567, 2002.

30. Wallner M, Herbst A, Behrens A, Crispin A, Stieber P, Göke B, Lamerz R and Kolligs FT: Methylation of serum DNA is an independent prognostic marker in colorectal cancer. Clin Cancer Res 12: 7347-7352, 2006.

31. Leung WK, To KF, Man EP, Chan MW, Bai AH, Hui AJ, Chan FK and Sung JJ: Quantitative detection of promoter hypermethylation in multiple genes in the serum of patients with colorectal cancer. Am J Gastroenterol 100: 2274-2279, 2005.
32. Herbst A, Rahmig K, Stieber P, Philipp A, Jung A, Ofner A, Crispin A, Neumann J, Lamerz R and Kolligs FT: Methylation of NEUROG1 in serum is a sensitive marker for the detection of early colorectal cancer. Am J Gastroenterol 106: 1110-1118, 2011.

33. Philipp AB, Stieber P, Nagel D, Neumann J, Spelsberg F, Jung A, Lamerz R, Herbst A and Kolligs FT: Prognostic role of methylated free circulating DNA in colorectal cancer. Int $\mathrm{J}$ Cancer 131: 2308-2319, 2012.

34. Cheng CK, Chan NP, Wan TS, Lam LY, Cheung CH, Wong TH, Ip RK, Wong RS and Ng MH: Helicase-like transcription factor is a RUNX1 target whose downregulation promotes genomic instability and correlates with complex cytogenetic features in acute myeloid leukemia. Haematologica 101: 448-457, 2016.

35. Caliskan C, Guler N, Karaca C, Makay O, Firat O and Korkut MA Negative prognostic factors in colorectal carcinoma: An analysis of 448 patients. Indian J Surg 72: 243-248, 2010.

36. Bosch SL, Teerenstra S, de Wilt JH, Cunningham $\mathrm{C}$ and Nagtegaal ID: Predicting lymph node metastasis in pT1 colorectal cancer: A systematic review of risk factors providing rationale for therapy decisions. Endoscopy 45: 827-834, 2013.

37. Leake I: Colorectal cancer. Understanding the routes of metastasis in colorectal cancer. Nat Rev Gastroenterol Hepatol 11: 270, 2014

38. Helmer RA, Foreman O, Dertien JS, Panchoo M, Bhakta SM and Chilton BS: Role of helicase-like transcription factor (hltf) in the G2/m transition and apoptosis in brain. PLoS One 8: e66799, 2013.

39. Huang D, Sun W, Zhou Y, Li P, Chen F, Chen H, Xia D, Xu E, Lai M, Wu Y, et al: Mutations of key driver genes in colorectal cancer progression and metastasis. Cancer Metastasis Rev 37: 173-187, 2018.

40. Chandrasinghe P, Cereser B, Moorghen M, Al Bakir I, Tabassum N, Hart A, Stebbing J and Warusavitarne J: Role of SMAD proteins in colitis-associated cancer: From known to the unknown. Oncogene 37: 1-7, 2018

41. $\mathrm{Xu} \mathrm{Y}$ and Pasche B: TGF- $\beta$ signaling alterations and susceptibility to colorectal cancer. Hum Mol Genet 16R: R14-R20, 2007.

42. Coates RF, Gardner JA, Gao Y, Cortright VM, Mitchell JM, Ashikaga T, Skelly J and Yang MX: Significance of positive and inhibitory regulators in the TGF- $\beta$ signaling pathway in colorectal cancers. Hum Pathol 66: 34-39, 2017.

43. Itatani $Y$, Kawada K, Fujishita T, Kakizaki F, Hirai H, Matsumoto T, Iwamoto M, Inamoto S, Hatano E, Hasegawa S, et al: Loss of SMAD4 from colorectal cancer cells promotes CCL15 expression to recruit CCR $1^{+}$myeloid cells and facilitate liver metastasis. Gastroenterology 145: 1064-1075.e11, 2013.

44. Voorneveld PW, Kodach LL, Jacobs RJ, Liv N, Zonnevylle AC, Hoogenboom JP, Biemond I, Verspaget HW, Hommes DW, de Rooij K, et al: Loss of SMAD4 alters BMP signaling to promote colorectal cancer cell metastasis via activation of Rho and ROCK. Gastroenterology 147: 196-208.e13, 2014.

45. Isaksson-Mettävainio M, Palmqvist R, Dahlin AM, Van Guelpen B, Rutegård J, Öberg A and Henriksson ML: High SMAD4 levels appear in microsatellite instability and hypermethylated colon cancers, and indicate a better prognosis. Int $\mathrm{J}$ Cancer 131: 779-788, 2012. 